Pacific Journal of Mathematics

BEST APPROXIMATION PROBLEMS IN TENSOR-PRODUCT 


\title{
BEST APPROXIMATION PROBLEMS IN TENSOR-PRODUCT SPACES
}

\author{
J. R. Respess, JR. AND E. W. Cheney
}

This paper concerns an existence problem for best approximations of bivariate functions. The approximating functions are taken from infinite-dimensional subspaces having tensor product form. Problems of this type arise, for example, in approximating the kernel of an integral equation by a degenerate ("separable") kernel. A sample of our results is this: let $G$ and $H$ be finite-dimensional subspaces in continuous function spaces $C(S)$ and $C(T)$ respectively. If one of these subspaces has a continuous proximity map and the other a Lipschitzian proximity map, then $G \otimes C(T)+C(S) \otimes H$ is proximinal in $C(S \times T)$; i.e., best approximations exist in this subspace.

Practical problems in numerical analysis, especially in solving two-point boundary value problems or integral equations, often require the approximation of a bivariate function by a combination of univariate functions. For example, if $f(s, t)$ is defined for $s \in S$ and $t \in T$, an approximation to $f$ of the following form may be required:

$$
f(s, t) \approx \sum_{i=1}^{m} x_{i}(s) h_{i}(t)+\sum_{i=1}^{n} y_{i}(t) g_{i}(s) .
$$

Here the base functions $g_{i}$ and $h_{i}$ are prescribed, and the coefficient functions $x_{i}$ and $y_{i}$ are at our disposal.

The problem of finding a best uniform approximation of the form (1) when all the functions involved are continuous is a difficult one, the difficulties being both theoretical and algorithmic. In the special case $n=m=1$, with $g_{1}(s)=h_{1}(t)=1$, the problem reduces to finding $x \in C(S)$ and $y \in C(T)$ which minimize the expression

$$
\|f-x-y\|=\sup \sup _{t}|f(s, t)-x(s)-y(t)| .
$$

The existence of minimizing pairs $(x, y)$ and an efficient algorithm for determining one of them were established by Diliberto and Straus [3]. See also $[1,8,5,10,9]$ for later work.

The general case of best approximation in (1) with uniform norm remains open. In this paper, the existence of optimal solutions to problem (1) is studied. Ideally, we would like to have constructive proofs of existence, but in general the available proofs are nonconstructive. 
If $U$ is a linear subspace of a normed space $X$, the distance from $x$ to $U$ is

$$
\operatorname{dist}(x, U)=\inf _{u \in U}\|x-u\| .
$$

If the infimum in (3) is attained for each $x \in X$, then the subspace $U$ is said to be proximinal. A mapping $A: X \rightarrow U$ such that $\|x-A x\|=\operatorname{dist}(x, U)$ for all $x$ is called a proximity map for $U$. Every proximinal subspace has a proximity map, but not necessarily a continuous one.

The following result from [13, p. 130] will be useful:

THEOREM 1. If $U, V$ and $U+V$, are closed subspaces of a Banach space, then there is a constant $c$ such that each element of $U+V$ is expressible as $u+v$ with $u \in U, v \in V$, and $\|u\|+\|v\| \leqq c\|u+v\|$.

TheOREM 2. For a pair of closed subspaces $U$ and $V$ in a Banach space the following properties are equivalent:

(1) $U+V$ is closed

(2) $U^{\perp}+V^{\perp}$ is closed

(3) $U^{\perp}+V^{\perp}$ is weak*-closed

(4) $U^{\perp}+V^{\perp}$ is proximinal.

Proof. The implication $1 \Rightarrow 3$ is proved as follows. H. Reiter showed in [12] that if $U, V$, and $U+V$ are closed subspaces in a Banach space, then $U^{\perp}+V^{\perp}=(U \cap V)^{\perp}$. Since the annihilator of a subspace is weak*-closed, [13, p. 91], $1 \Rightarrow 3$. The implication $3 \Rightarrow 4$ is an observation made by Phelps [11]. The implication $4 \Rightarrow 2$ is trivial, since every proximinal set is closed. The implication $2 \Rightarrow 1$ is another result of Reiter [12].

THEOREM 3. Let $U$ and $V$ be weak*-closed subspaces in a conjugate Banach space $X^{*}$. If $U+V$ is norm closed, then it is weak*closed and proximinal.

Proof. Since $U$ and $V$ are weak*-closed, they satisfy $U=\left(U_{\perp}\right)^{\perp}$ and $V=\left(V_{\perp}\right)^{\perp}$, where $U_{\perp}=\{x \in X:\langle x, u\rangle=0$ for all $u \in U\}$. By Theorem 2 (in particular the implications $2 \Rightarrow 3 \Rightarrow 4$ ), our conclusion follows.

TheOREM 4. Let $U$ and $V$ be subspaces in a normed space $X$. Assume that $U$ is proximinal, and that for each $x \in X$ there corresponds a weakly compact set $K(x) \subset V$ with the property

$$
\inf _{v \in K(x)} \operatorname{dist}(x-v, U)=\inf _{v \in V} \operatorname{dist}(x-v, U) .
$$


Then $U+V$ is proximinal.

Proof. By the Hahn-Banach theorem,

$$
\operatorname{dist}(x, U)=\sup \left\{\langle\phi, x\rangle: \phi \in U^{\perp},\|\phi\|=1\right\} .
$$

This shows that the function $x \mapsto \operatorname{dist}(x, U)$ is weakly lower-semicontinuous, since it is the supremum of a family of weakly continuous functions. Therefore, if $x$ is fixed, the expression dist $(x-v, U)$ will attain its infimum at some point $v^{\prime} \in K(x)$. Select $u^{\prime} \in U$ as a best approximation to $x-v^{\prime}$. Then for any $v \in V$ and $u \in U$ we have $\left\|x-u^{\prime}-v^{\prime}\right\|=\operatorname{dist}\left(x-v^{\prime}, U\right) \leqq \operatorname{dist}(x-v, U) \leqq\|x-v-u\|$.

THEOREM 5. Let $U$ and $V$ be proximinal subspaces in a Banach space $X$. Assume that $U+V$ is closed, and that $V$ has a proximity map $A$ such that for each $c \in X$, the map $u \mapsto A(c-u)$ is weakly compact from $U$ into $V$. Then $U+V$ is proximinal.

Proof. Let $c$ be any element of $X$, and select $z_{n} \in U+V$ so that $\left\|c-z_{n}\right\| \rightarrow \operatorname{dist}(c, U+V)$. The sequence $\left\{z_{n}\right\}$ is bounded. Since $U+V$ is closed, Theorem 1 implies that $z_{n}$ can be expressed as $u_{n}+v_{n}$ with $u_{n} \in U, v_{n} \in V$, and $\left\{u_{n}\right\}$ bounded. Put $v_{n}^{\prime}=A\left(c-u_{n}\right)$. Since $\left\{u_{n}\right\}$ is bounded, $\left\{v_{n}^{\prime}\right\}$ lies in a weakly compact subset $K$ of $V$. Then for each $n$,

Hence

$$
\begin{aligned}
\inf _{v \in K} \operatorname{dist}(c-v, U) & \leqq \operatorname{dist}\left(c-v_{n}^{\prime}, U\right) \leqq\left\|c-u_{n}-v_{n}^{\prime}\right\| \\
& \leqq\left\|c-u_{n}-v_{n}\right\| .
\end{aligned}
$$

$$
\inf _{v \in K} \operatorname{dist}(c-v, U) \leqq \operatorname{dist}(c, U+V)=\inf _{v \in V} \operatorname{dist}(c-v, U) .
$$

Thus Theorem 4 is applicable, and $U+V$ is proximinal.

The uncompleted tensor product of two normed spaces $X$ and $Y$ is the set of all finite sums of the form $\sum x_{i} \otimes y_{i}$ with $x_{i} \in X$ and $y_{i} \in Y$. An equivalence relation is introduced by stipulating that $\sum x_{i} \otimes y_{i}$ is (equivalent to) 0 when $\sum\left\langle f, x_{i}\right\rangle y_{i}=0$ for all $f \in X^{*}$.

A norm $\alpha$ on $X \otimes Y$ is termed a cross-norm if $\alpha(x \otimes y)=\|x\|\|y\|$ for all $x \in X$ and all $y \in Y$. A cross-norm $\alpha$ is said to be a uniform cross-norm if

$$
\alpha\left(\sum A x_{i} \otimes B y_{i}\right) \leqq\|A\|\|B\| \alpha\left(\sum x_{i} \otimes y_{i}\right)
$$

for any bounded linear operators $A$ and $B$.

The completion of the normed linear space $X \otimes Y$ with a cross- 
norm $\alpha$ is denoted here by $X \otimes_{\alpha} Y$. For other matters concerning tensor products, see Schatten [14], Gilbert and Leih [9], or Diestel and Uhl [2]. In particular, we use the isometric identification $\mathscr{L}\left(X, Y^{*}\right)=\left(X \otimes_{r} Y\right)^{*}[14$, p. 47].

The following theorem resulted from discussions with Professor John E. Gilbert, to whom we are indebted.

Theorem 6. Let $G$ and $H$ be complemented subspaces in Banach spaces $X$ and $Y$ respectively. For any uniform cross-norm $\alpha$, $\left(G \otimes_{\alpha} Y\right)+\left(X \otimes_{\alpha} H\right)$ is complemented (and therefore closed) in $X \otimes_{\alpha} Y$.

Proof. Let $P$ be a (bounded linear) projection of $X$ onto $G$. Define $P^{\prime}$ on the uncompleted tensor product $X \otimes Y$ by putting $P^{\prime}\left(\sum x_{i} \otimes\right.$ $\left.y_{i}\right)=\sum P x_{i} \otimes y_{i}$. By the uniform property of the cross-norm $\alpha$, we have $\alpha\left[P^{\prime}\left(\sum x_{i} \otimes y_{i}\right)\right] \leqq\|P\| \alpha\left(\sum x_{i} \otimes y_{i}\right)$. Thus $P^{\prime}$ is uniformly continuous on a dense subset of $X \otimes_{\alpha} Y$ and has therefore a unique continuous extension to $X \otimes_{\alpha} Y$. Thus extended, $P^{\prime}$ is a projection of $X \otimes_{\alpha} Y$ onto $G \otimes_{\alpha} Y$. In the same way, starting with a projection $Q$ of $Y$ onto $H$ we define a projection $Q^{\prime}$ of $X \otimes_{\alpha} Y$ onto $X \otimes_{\alpha} H$. One verifies easily that $P^{\prime}$ commutes with $Q^{\prime}$. Hence [see 4, p. 481] $P^{\prime}+Q^{\prime}-P^{\prime} Q^{\prime}$ is a projection of $X \otimes_{\alpha} Y$ onto $\left(G \otimes_{\alpha} Y\right)+\left(X \otimes_{\alpha} H\right)$.

In the following discussion, $T$ will denote an arbitrary compact Hausdorff space. Then $C(T)$ is the usual space of continuous realvalued functions on $T$.

The special cross-norm $\lambda$ is defined by the equation

$$
\lambda\left(\sum x_{i} \otimes y_{i}\right)=\sup _{f}\left\|\Sigma\left\langle f, x_{i}\right\rangle y_{i}\right\|
$$

where $f$ ranges over the unit cell in $X^{*}$.

The next theorem has been given in [6]; the proof is included because it is brief.

THEOREM 7. If there exists a continuous proximity map from the Banach space $X$ onto a subspace $G$, then $C(T) \otimes_{\lambda} G$ is proximinal in $C(T) \otimes_{2} X$.

Proof. By a theorem of Grothendieck, [15, p. 357], $C(T) \otimes_{\lambda} X$ is isometric with $C(T, X)$. The latter is the Banach space of all continuous maps $f$ from $T$ into $X$, normed by putting $\|f\|=$ $\sup _{t}\|f(t)\|_{X}$. If $A$ is a continuous proximity map from $X$ onto $G$ then let $A^{\prime}$ be defined from $C(T, X)$ onto $C(T, G)$ by the equation $A^{\prime} f=A \circ f$. It is elementary to prove that $A^{\prime}$ is a continuous proximity map. 
THEOREM 8. If $G$ is a subspace of $C(S)$ such that $G \otimes_{2} C(T)$ is proximinal in $C(S \times T)$, then $G$ is proximinal.

Proof. Assume that $G \otimes_{\lambda} C(T)$ is proximinal. Let $x$ be any element of $C(S)$. Put $x^{\prime}(x, t)=x(s)$ for all $(s, t) \in S \times T$. Note that for any $g \in G$,

$$
\operatorname{dist}\left(x^{\prime}, G \otimes{ }_{2} C(T)\right) \leqq\left\|x^{\prime}-g \otimes 1\right\|=\|x-g\|
$$

whence $\operatorname{dist}\left(x^{\prime}, G \otimes_{\lambda} C(T)\right) \leqq \operatorname{dist}(x, G)$. Let $z$ be a best approximation to $x^{\prime}$ from $G \otimes_{\lambda} C(T)$. Select $\tau \in T$ so that $\left\|x^{\prime}-z\right\|=$ $\sup _{s}\left|x^{\prime}(s, \tau)-z(s, \tau)\right|$. Put $g(s)=z(s, \tau)$. Then $g \in G$, and $g$ is a best approximation to $x$ since

$$
\begin{aligned}
\|x-g\| & =\sup _{s}|x(s)-g(s)|=\sup _{s}\left|x^{\prime}(s, \tau)-z(s, \tau)\right|=\left\|x^{\prime}-z\right\| \\
& =\operatorname{dist}\left(x^{\prime}, G \otimes_{\lambda} C(T)\right) \leqq \operatorname{dist}(x, G) .
\end{aligned}
$$

The following result is called "The Sitting-Duck Theorem" because it is thought to be true under weaker hypotheses on $H$, and is therefore vulnerable to generalization.

THEOREM 10 ("Sitting Duck"). Let G be a finite-dimensional subspace of $C(S)$ with a continuous proximity map. Let $H$ be a finitedimensional subspace of $C(T)$ with a Lipschitzian proximity map. Then $G \otimes C(T)+C(S) \otimes H$ is complemented and proximinal in $C(S \times T)$.

Proof. By Theorem 7, the subspaces $U=G \otimes C(T)$ and $V=$ $C(S) \otimes H$ are proximinal. By Theorem $6, U+V$ is complemented and closed. Let $A$ be a Lipschitzian proximity map of $C(T)$ onto $H$, and put $\left(A^{\prime} f\right)(s, t)=\left(A f_{s}\right)(t)$. Then $A^{\prime}$ is a proximity map of $C(S \times T)$ onto $V$. Define $\Gamma: U \rightarrow V$ by $\Gamma u=A^{\prime}(f-u)$, where $f$ is now fixed. By the following lemma, $\Gamma$ is compact. By Theorem 5, $U+V$ is proximinal. (Note: $f_{s}(t)=f^{t}(s)=f(s, t)$.)

REMARK. Instead of assuming that $G$ has a continuous proximity map, we can assume that $G \otimes C(T)$ is proximinal in $C(S \times T)$.

Lemma. The map $\Gamma: U \rightarrow V$ defined in the proof of Theorem 10 is compact.

Proof. Let $B=\{u \in U:\|u\| \leqq k\}$. We will show that $\Gamma(B)$ has compact closure in $V$. By the Ascoli theorem, it suffices to show that $\Gamma(B)$ is bounded and equicontinuous. 
If $u \in U$ then $\|\Gamma(u)\|=\left\|A^{\prime}(f-u)\right\| \leqq 2\|f-u\| \leqq 2\|f\|+2 k$. Hence $\Gamma(B)$ is bounded. The remainder of the proof addresses the equicontinuity. Assume that $\|A x-A y\| \leqq \lambda\|x-y\|$ for $x, y \in C(T)$. Let $n$ denote the dimension of $G$.

Select $\left\{g_{1}, \cdots, g_{n}\right\} \subset G$ and $\left\{\phi_{1}, \cdots, \phi_{n}\right\} \subset C(S)^{*}$ so that $\left\langle\dot{\phi}_{i}, g_{j}\right\rangle=\delta_{i j}$, $\left\|g_{i}\right\|=\left\|\dot{\phi}_{i}\right\|=1$ ("biorthonormality"). If $u(s, t)=\sum_{i=1}^{n} x_{i}(t) g_{i}(s)$ then

$$
\left|x_{i}(t)\right|=\left|\left\langle\phi_{i}, u^{t}\right\rangle\right| \leqq\left\|u^{t}\right\| \leqq k .
$$

Let $\left(s_{0}, t_{0}\right)$ be a point of $S \times T$ at which equicontinuity is to be proved. Let $\varepsilon>0$. By the equicontinuity of the unit cell in $G$ there is a neighborhood $N_{1}$ of $s_{0}$ such that for all $s \in N_{1}$ and for all $g \in G$, $\left|g(s)-g\left(s_{0}\right)\right| \leqq \varepsilon\|g\|$. Similarly, there is a neighborhood $N_{2}$ of $t_{0}$ such that for all $t \in N_{2}$ and for all $h \in H,\left|h(t)-h\left(t_{0}\right)\right|<\varepsilon\|h\|$. By the equicontinuity of $\left\{f^{t}: t \in T\right\}$ we can shrink the neighborhood $N_{1}$ if necessary so that $\left|f^{t}(s)-f^{t}\left(s_{0}\right)\right|<\varepsilon$ for all $s \in N_{1}$ and all $t \in T$. Then $\left\|f_{s}-f_{s_{0}}\right\|<\varepsilon$. Let $N=N_{1} \times N_{2}$. If $(s, t) \in N$ then

$$
\begin{aligned}
& \left|(\Gamma u)(s, t)-(\Gamma u)\left(s_{0}, t_{0}\right)\right| \\
& \quad \leqq\left|(\Gamma u)(s, t)-(\Gamma u)\left(s_{0}, t\right)\right|+\left|(\Gamma u)\left(s_{0}, t\right)-(\Gamma u)\left(s_{0}, t_{0}\right)\right| \\
& \quad=\left|A\left(f_{s}-u_{s}\right)(t)-A\left(f_{s_{0}}-u_{s_{0}}\right)(t)\right|+\left|A\left(f_{s_{0}}-u_{s_{0}}\right)(t)-A\left(f_{s_{0}}-u_{s_{0}}\right)\left(t_{0}\right)\right| \\
& \quad \leqq\left\|A\left(f_{s}-u_{s}\right)-A\left(f_{s_{0}}-u_{s_{0}}\right)\right\|+\left\|A\left(f_{s_{0}}-u_{s_{0}}\right)\right\| \varepsilon \\
& \quad \leqq \lambda\left\|\left(f_{s}-u_{s}\right)-\left(f_{s_{0}}-u_{s_{0}}\right)\right\|+2\left\|f_{s_{0}}-u_{s_{0}}\right\| \varepsilon \\
& \quad \leqq \lambda\left\{\left\|f_{s}-f_{s_{0}}\right\|+\left\|u_{s}-u_{s_{0}}\right\|\right\}+2\{\|f\|+\|u\|\} \varepsilon \\
& \quad \leqq \lambda\left\{\varepsilon+\left\|\sum\left[g_{i}(s)-g_{i}\left(s_{0}\right)\right] x_{i}\right\|\right\}+2\|f\| \varepsilon+2 k \varepsilon \\
& \quad \leqq \lambda\{\varepsilon+n \varepsilon k\}+2\|f\| \varepsilon+2 k \varepsilon .
\end{aligned}
$$

In a separate paper, we have given examples of subspaces having Lipschitzian proximity maps in a space $C(T)$. These can be of any finite dimension or infinite dimensional. The situation is rather complicated, however, and the topological structure of $T$ must be taken into account.

In several of the following theorems we require the equation

$$
X^{*} \otimes_{\lambda} Y^{*} \subset \mathscr{L}\left(X, Y^{*}\right)=\left(X \otimes_{r} Y\right)^{*} \text {. }
$$

The identifications made here are as follows. With an element $\sum \varphi_{i} \otimes \psi_{i}$ in $X^{*} \otimes Y^{*}$ (uncompleted tensor product) we associate an operator $A \in \mathscr{L}\left(X, Y^{*}\right)$ whose defining equation is $A x=\sum\left\langle\varphi_{i}, x\right\rangle \psi_{i}$. With an arbitrary operator $B$ in $\mathscr{L}\left(X, Y^{*}\right)$ we associate a functional $\Phi$ in $\left(X \otimes_{r} Y\right)^{*}$ by putting $\Phi\left(\sum x_{i} \otimes y_{i}\right)=\sum\left\langle B x_{i}, y_{i}\right\rangle$.

The weak*-topology in $\mathscr{L}\left(X, Y^{*}\right)$ is the weak topology induced by the duality of $X \otimes_{r} Y$ with $\left(X \otimes_{r} Y\right)^{*}$. Convergence of a net $A_{\alpha}$ to 0 in this topology means $\left\langle A_{\alpha} x, y\right\rangle \rightarrow 0$ for all $x \in X$ and $y \in Y$. This topology is also called the weak*-operator topology. 
THeOREm 17. Let $P$ be a projection on a Banach space $X$. Let $Y$ be any other Banach space. Then the subspace

$$
M=\left\{A \circ P: A \in \mathscr{L}\left(X, Y^{*}\right)\right\}
$$

is complemented, weak*-closed, and hence proximinal in $\mathscr{L}\left(X, Y^{*}\right)$.

Proof. For $A \in \mathscr{L}\left(X, Y^{*}\right)$, define $p(A)=A \circ P$. Then $p$ is a bounded linear mapping of $\mathscr{L}\left(X, Y^{*}\right)$ into $M$. Since $p(A \circ P)=$ $A \circ P \circ P=A \circ P$, it follows that $p$ acts like the identity on $M$. Therefore $p$ is a projection and $M$ is complemented.

In order to show that $M$ is weak*-closed, we note first that $M$ is the null-space of $i-p$, where $i$ denotes the identity map on $\mathscr{L}\left(X, Y^{*}\right)$. Next we observe that $p$ (and hence $i-p$ ) is weak*continuous. Indeed, if $A_{\alpha}$ is a net in $\mathscr{L}\left(X, Y^{*}\right)$ which converges in the weak*- topology to 0 , then $\left\langle A_{\alpha} x, y\right\rangle \rightarrow 0$ for all $(x, y) \in X \times Y$. Hence $\left\langle p\left(A_{\alpha}\right) x, y\right\rangle=\left\langle A_{\alpha} P x, y\right\rangle \rightarrow 0$ for all $(x, y)$, and $p\left(A_{\alpha}\right)$ converges to 0 in the weak*-topology.

A completely analogous proof establishes the next result.

THeOREM 18. Let $Q$ be a projection on a Banach space $Y$. Let $X$ be any other Banach space. Then the subspace

$$
N=\left\{Q^{*} \circ A: A \in \mathscr{L}\left(X, Y^{*}\right)\right\}
$$

is complemented, weak*-closed and hence proximinal in $\mathscr{L}\left(X, Y^{*}\right)$.

THEOREM 19. Let $P$ and $Q$ be projections on Banach spaces $X$ and $Y$ respectively. Then

$$
\left\{A \circ P+Q^{*} \circ B: A, B \in \mathscr{L}\left(X, Y^{*}\right)\right\}
$$

is complemented, weak*-closed, and proximinal in $\mathscr{L}\left(X, Y^{*}\right)$.

Proof. It is sufficient to verify that the projections $p$ and $q$ defined by $p(A)=A \circ P$ and $q(A)=Q^{*} \circ A$ commute with each other. But this is obviously true:

$$
p(q(A))=\left(Q^{*} \circ A\right) \circ P=Q^{*} \circ(A \circ P)=q(p(A)) .
$$

REMARKs. Theorem 19 was suggested to us by an anonymous referee for the Mathematical Proceedings of the Cambridge Philosophical Society. We had, prior to his suggestion, established only the following theorem by a different argument.

TheOREM 20. Let $G$ and $H$ be finite-dimensional subspaces in 
conjugate Banach spaces $X^{*}$ and $Y^{*}$ respectively. Then $G \otimes Y^{*}+$ $X^{*} \otimes H$ is complemented, weak*-closed, and proximinal in $\mathscr{L}\left(X, Y^{*}\right)$. It is therefore complemented and proximinal in $X^{*} \otimes_{\lambda} Y^{*}$.

Proof. We prove first that $G \otimes Y^{*}=\left\{A \circ P: A \in \mathscr{L}\left(X, Y^{*}\right)\right\}$ for an appropriate projection $P: X \rightarrow X$. Indeed, select a basis $\left\{g_{1}, \cdots, g_{n}\right\}$ for $G$ and then select $x_{1}, \cdots, x_{n}$ in $X$ so that $\left\langle g_{i}, x_{j}\right\rangle=\delta_{i j}$. Put $P x=\sum\left\langle x, g_{i}\right\rangle x_{i}$. If $\sum g_{i} \otimes \psi_{i}$ is any element of $G \otimes Y^{*}$, let $A$ be an element of $\mathscr{L}\left(X, Y^{*}\right)$ such that $A x_{i}=\psi_{i}$. Then $A \circ P=\sum g_{i} \otimes \psi_{i}$. Conversely, if $A \in \mathscr{L}\left(X, Y^{*}\right)$, then $A \circ P=\sum g_{i} \otimes A x_{i} \in G \otimes Y^{*}$.

A similar argument applies to $X^{*} \otimes H$, and then Theorem 19 establishes the desired conclusion.

In approximation problems, it is a fortunate circumstance when a subspace of functions being used as approximants has a linear proximity map. Of course, this is the rule in Hilbert space, but the exception in other spaces, although proximinal hyperplanes always have linear proximity maps in any normed space. In spaces $C(T)$, a finite-dimensional subspace can have a linear proximity map, but if this happens, $T$ must possess isolated points.

If a proximity map $P$ from a normed space $X$ onto a subspace $V$ is linear, then $P$ is a projection (i.e., a bounded, linear, idempotent, surjective map.) It is elementary to prove that for a projection $P$ the properties of being a proximity map and satisfying the equation $\|I-P\|=1$ are equivalent.

Another elementary result is that if $P$ and $Q$ are projections on a normed space $X$, and if $Q P=P Q P$, then $P+Q-P Q$ is a projection onto the vector sum of the ranges of $P$ and $Q$. This vector sum must then be complemented and closed. We can now prove:

THEOREM 21. If $P$ and $Q$ are linear proximity maps, then the same is true of the Boolean sum $P+Q-P Q$, provided that $P Q P=Q P$.

Proof. It is only necessary to verify that $\|I-P-Q+P Q\|=1$. This follows from writing the operator in question in the form $(I-P)(I-Q)$.

Lemma. If $A \in \mathscr{L}(X, X)$ and $B \in \mathscr{L}(Y, Y)$ then the operator $A \otimes B$ defined on $X \otimes Y$ by the equation

$$
(A \otimes B) \sum x_{i} \otimes y_{i}=\sum A x_{i} \otimes B y_{i}
$$

has a unique extension $A \otimes_{\alpha} B$ in $\mathscr{L}\left(X \otimes_{\alpha} Y, X \otimes_{\alpha} Y\right)$, for any uniform cross norm $\alpha$. 
THEOREM 22. If $G$ and $H$ are subspaces having linear proximity maps in Banach spaces $X$ and $Y$ respectively, then $G \otimes_{\alpha} Y+X \otimes_{\alpha} H$ is proximinal in $X \otimes_{\alpha} Y$, for any uniform cross-norm $\alpha$.

Proof. Suppose that $P: X \rightarrow G$ and $Q: Y \rightarrow H$ are linear proximity maps. Then $P \otimes_{\alpha} I_{Y}$ and $I_{X} \otimes_{\alpha} Q$ are linear proximity maps from $X \otimes_{\alpha} Y$ onto $G \otimes_{\alpha} Y$ and $X \otimes_{\alpha} H$, respectively. They commute, by the lemma which follows. Hence by the preceding theorem, their Boolean sum is a linear proximity map. Its range is the sum of the ranges of the constituent maps, i.e., $G \otimes_{\alpha} Y+X \otimes_{\alpha} H$.

Lemma. Let $A_{1}$ and $A_{2}$ be commuting elements of $\mathscr{L}(X, X)$. Let $B_{1}$ and $B_{2}$ be commuting elements of $\mathscr{L}(Y, Y)$. Then $A_{1} \otimes_{\alpha} B_{1}$ commutes with $A_{2} \otimes_{\alpha} B_{2}$ for any uniform cross-norm $\alpha$.

\section{REFERENCES}

1. G. Aumann, Uber approximative Nomographie II, Bayer. Akad. Wiss. Math. Nat. Kl. S.B., (1959), 103-109.

2. J. Diestel and J. J. Uhl, Vector measures, Mathematical Surveys, no. 15, Amer. Math. Soc., 1977.

3. S. P. Diliberto and E. G. Straus, On the approximation of a function of several variables by the sum of functions of fewer variables, Pacific J. Math., 1 (1951), 195-210. 4. N. Dunford and J. T. Schwartz, Linear Operators, Part I, Interscience Publishers, New York, 1958.

5. N. Dyn, A straightforward generalization of Diliberto and Straus' algorithm does not work, Math. Research Center, Madison, Wisc., Dec. 1978. J. Approximation Theory, 30 (1980), 247-250.

6. C. Franchetti and E. W. Cheney, Best approximation problems for multivariate functions, Report 155, Center for Numerical Analysis, The University of Texas, January 1980. Boll. Unione Mat. Ital., 18-B (1981), 1003-1015.

7. J. E. Gilbert and T. J. Leih, Factorization, tensor products, and bilinear forms in Banach space theory, in Notes in Banach Spaces, H. E. Lacey, ed., The University of Texas Press, Austin, 1980, 182-305.

8. M. von Golitschek and E. W. Cheney, On the algorithm of Diliberto and Straus for approximating bivariate functions by univariate ones, Numer. Funct. Analysis and Optimization, 1 (1979), 341-363.

9. C. T. Kelley, A note on the approximation of functions of several variables by sums of functions of one variable, Math. Research Center, Madison, Wisc., Report No. 1873, (1978). J. Approximation Theory, 29 (1980), 305-322.

10. W. A. Light and E. W. Cheney, On the approximation of a bivariate function by the sum of univariate functions, Center for Numerical Analysis, The University of Texas, Report 140, 1978. J. Approximation Theory.

11. R. R. Phelps, Uniqueness of Hahn-Banach extensions and unique best approximation, Trans. Amer. Math. Soc., 95 (1960), 238-255.

12. H. Reiter, Contributions to harmonic analysis, VI, Ann. of Math., 77 (1963), $552-562$.

13. W. Rudin, Functional Analysis, McGraw-Hill Book Co., New York, 1973.

14. R. Schatten, $A$ theory of cross-spaces, Annals of Mathematics Studies, Number 26, Princeton University Press, 1950. 
15. Z. Semadeni, Banach spaces of continuous functions, Polish Scientific Publishers, Warsaw, 1971.

Received June 18, 1981. This work was supported by the U.S. Army Research Office under grant DAAG 29-80-K-0039.

UNIVERSITY OF TEXAS

AUSTIN, TX 78712 


\section{PACIFIC JOURNAL OF MATHEMATICS}

\section{EDITORS}

DONALD BABBITT (Managing Editor)

University of California

Los Angeles, CA 90024

Hugo RossI

University of Utah

Salt Lake City, UT 84112

C. C. MOORE and ARThur Agus

University of California

Berkeley, CA 94720
J. DugundJI

Department of Mathematics

University of Southern California

Los Angeles, CA 90007

R. FINN and J. MILGRAM

Stanford University

Stanford, CA 94305

\section{ASSOCIATE EDITORS}
R. ARENS
E. F. BeCKENBACH
B. H. NeumanN
F. WOLF
K. YoSHIDA

\section{SUPPORTING INSTITUTIONS}

UNIVERSITY OF ARIZONA

UNIVERSITY OF BRITISH COLUMBIA

CALIFORNIA INSTITUTE OF TECHNOLOGY

UNIVERSITY OF CALIFORNIA

MONTANA STATE UNIVERSITY

UNIVERSITY OF NEVADA, RENO

NEW MEXICO STATE UNIVERSITY

OREGON STATE UNIVERSITY

\author{
UNIVERSITY OF OREGON \\ UNIVERSITY OF SOUTHERN CALIFORNIA \\ STANFORD UNIVERSITY \\ UNIVERSITY OF AAWAII \\ UNIVERSITY OF TOKYO \\ UNIVERSITY OF UTAH \\ WASHINGTON STATE UNIVERSITY \\ UNIVERSITY OF WASHINGTON
}

The Supporting Institutions listed above contribute to the cost of publication of this Journal, but they are not owners or publishers and have no responsibility for its content or policies,

Mathematical parers intended for publication in the Pacific Journal of Mathematics should be in typed form or offset-reproduced, (not dittoed), double spaced with large margins. Please do not use built up fractions in the text of the manuscript. However, you may use them in the displayed equations. Underline Greek letters in red, German in green, and script in blue. The first paragraph or two must be capable of being used separately as a synopsis of the entire paper. Please propose a heading for the odd unmbered pages of less than 35 characters. Manuscripts, in triplicate, may be sent to any one of the editors. Please classify according to the scheme of Math. Reviews, Index to Vol. 39. Supply name and address of author to whom proofs should be sent. All other communications should be addressed to the managing editor, or Elaine Barth, University of California, Los Angeles, California, 90024.

50 reprints to each author are provided free for each article, only if page charges have been substantially paid. Additional copies may be obtained at cost in multiples of 50 .

The Pacific Journal of Mathematics is issued monthly as of January 1966, Regular subscription rate: $\$ 114.00$ a year (6 Vol., 12 issues). Special rate: $\$ 57.00$ a year to individual members of supporting institution.

Subscriptions, orders for numbers issued in the last three calendar years, and changes of address shoud be sent to Pacific Journal of Mathematics, P.O. Box 969, Carmel Valley, CA 93924, U.S.A. Old back numbers obtainable from Kraus Periodicals Co., Route 100, Millwood, NY 10546.

\section{PUBLISHED BY PACIFIC JOURNAL OF MATHEMATICS, A NON-PROFIT CORPORATION}

Printed at Kokusai Bunken Insatsusha (International Academic Printing Co., Ltd.). 8-8, 3-chome, Takadanobaba, Shinjuku-ku, Tokyo 160, Japan. 


\section{Pacific Journal of Mathematics}

\section{Vol. 102, No. 2 \\ February, 1982}

Richard A. Boyce, Irreducible representations of finite groups of Lie type through block theory and special conjugacy classes ...............253

Robert Jay Daverman and Dennis J. Garity, Intrinsically

$(n-2)$-dimensional cellular decompositions of $E^{n} \ldots \ldots \ldots \ldots 275$

Juan Ferrera, Spaces of weakly continuous functions ................285

William George Frederick, $\mu$-theta functions ................... 293

Christopher George Gibson and T. D. Ward, On stratifying pairs of linear

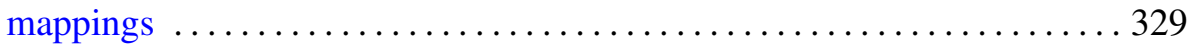

Stanley Joseph Gurak, Minimal polynomials for Gauss circulants and cyclotomic units ........................................ 347

Joachim Georg Hartung, On two-stage minimax problems ............. 355

Robert P. Kaufman, Hausdorff measure, BMO, and analytic functions . . . . 369

Neal I. Koblitz, $p$-adic analog of Heine's hypergeometric $q$-series . . . . . . . 373

Kurt Kreith, Picone-type theorems for hyperbolic partial differential

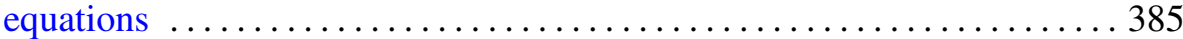

Nicholas J. Kuhn, The geometry of the James-Hopf maps ............. 397

Donald Michael Redmond, Explicit formulae for a class of Dirichlet

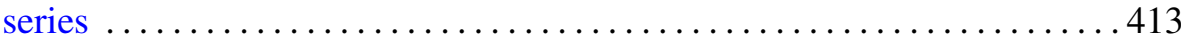

J. R. Respess and Elliott Ward Cheney, Jr., Best approximation problems in tensor-product spaces . .............................. 437

Allen Ross Schweinsberg, The operator equation $A X-X B=C$ with normal $A$ and $B$

Hans-Willi Siegberg and Guentcho Svetoslavov Skordev, Fixed point index and chain approximations

Kondagunta Sundaresan, Geometry and nonlinear analysis in Banach spaces 